\title{
Autoimmune inner ear disease - a case report and brief review of literature
}

\author{
Deepa Panikkath*, Avinash Adiga, and Michael Calmes \\ Department of Internal Medicine, Texas Tech University Health Sciences Center, Lubbock, Texas, USA
}

\begin{abstract}
Autoimmune inner ear disease (AIED) is a rare syndrome characterized by progressive bilateral sensorineural hearing loss (SNHL). It is a poorly understood form of hearing loss often accompanied by vestibular symptoms and can mimic Meniere's disease. Diagnosis is mainly based on clinical characteristics and response to immunosuppressive agents. It is one of the rare causes of hearing loss that is potentially reversible with treatment if rapidly initiated. We hereby present a case of a 54-year-old female diagnosed with Autoimmune inner ear disease and a brief review of literature.
\end{abstract}

\section{Introduction}

Autoimmune inner ear disease (AIED) is a relatively rare cause of sensorineural hearing loss probably accounting for less than $1 \%$ of hearing loss [1]. Initially described in 1979 by McCabe, it is characterized by rapid progressive hearing loss often occurring bilaterally with or without vestibular symptoms [2]. The pathophysiology of this disease still remains to be fully elucidated. Autoimmunity is thought to play a role based on the presence of autoantibodies directed against the inner ear, its association with other systemic autoimmune disorders, and treatment response to immunosuppressants. Due to lack of a definite diagnostic test, the clinical presentation and treatment response to immunosuppressants establishes its diagnosis.

\section{Case report}

A 54-year-old Caucasian female presented with complaints of sudden onset hearing difficulty and dizziness following a brief flu like illness. She had no accompanying tinnitus, headaches, blurry vision, numbness or weakness of extremities. Physical examination showed no neurological deficits but was significant for bilateral hearing loss. Her routine laboratory investigations were normal. MRI of the brain was within normal limits. Audiogram done for evaluation of hearing difficulty revealed asymmetric bilateral sensorineural deficit, right more than the left. Autoimmune workup was done which was positive for an elevated ANA with titer of 1:160 and a speckled pattern seen on immunofluorescence. She also had positive antibody against 68$\mathrm{kd}$ antigen. She was diagnosed with Autoimmune inner ear disease based on her clinical presentation and positive serology for anti-68kd antibodies. She was started on a trial of oral steroids which improved her vestibular symptoms. No significant improvement in hearing was seen. Her clinical course worsened with development of tinnitus. Repeat laboratory test showed persistence of anti- 68kd antibody and risingANA titers to 1:320. She was continued on daily oral steroids and methotrexate $10 \mathrm{mg}$ weekly dose was initiated. With this regime, she had dramatic improvement in her hearing deficit. Her steroids were then tapered to a low dose with addition of diuretics to her treatment regime. She continued to be in remission on this regime.

\section{Discussion}

The initial description of AIED was in 1979 by McCabe who described a series of patients with rapidly progressive bilateral sensorineural hearing loss (SNHL) [2]. The exact pathogenesis of AIED remains unclear. Autoimmunity was initially proposed by McCabe as the cause as patients showed marked improvement with steroids [2]. Inner ear is now considered to be an immunocompetent site with the endolymphatic sac and presence of resident $\mathrm{T}$ cells capable of mounting adequate immune responses $[3,4]$. A number of antibodies against various inner ear antigens have been identified in experimental and human AIED. Their exact pathogenic role is still unclear. These include antibodies against subcomponents of crude inner ear antigens like 31 $\mathrm{kD}$ protein and the $60 \mathrm{kD}$ protein, antibodies against cochlin, connexin 26 , myelin protein $\mathrm{P} 0$ etc $[5,6]$. One of the most studied antigenin AIED is the inner ear 68 -kd antigen. Autoantibodies against this antigen was initially detected in 1990 by Harris and Sharp in patients with idiopathic bilateral sensorineural hearing loss [7]. In 1994, Moscicki et al. confirmed these findings and demonstrated a correlation between steroid responsiveness and patient's $68-\mathrm{kD}$ antibody status [8].This 68$\mathrm{kd}$ antigen was later identified to be heat shock protein 70(hsp 70) [9]. Approximately $30 \%$ of patients with AIED also have coexisting systemic autoimmune disease like systemic lupus erythematosus, rheumatoid arthritis, disseminated vasculitis, Sjögren's syndrome, myasthenia gravis, Hashimoto's thyroiditis etc. [10,11]. The patient reported above demonstrated antibodies against $68-\mathrm{kD}$ antigen and ANA positivity in titers ranging from 1:160 to 1:320 in a speckled pattern. ANA positivity in a speckled pattern have been reported previously by Dayal et al. in patients with AIED [12].

The hallmark of AIED is the presence of rapidly progressive, often bilateral hearing loss over a period of weeks to months. Vestibular symptoms have been reported in almost $50 \%$ of patients. Due to

Correspondence to: Deepa Panikkath, Department of Internal Medicine, Texas Tech University Health Sciences Center, 3601 th street, Lubbock, Texas - 79430, USA, Tel: 806-418-1847, Fax: 806-743-3143, E-mail: deeparag@gmail.com

Received: May 20, 2016; Accepted: July 05, 2016; Published: July 08, 2016 
the constellation of auditory and vestibular symptoms, it can mimic Meniere's disease. The course often fluctuates with episodic flares and periods of remission $[2,13]$

Diagnosis is often based on clinical suspicion and improvement of symptoms with immunosuppressants due to lack of specific diagnostic tests. Western blot tests have been widely used to detect anti-68-kd antibodies in the sera. A recent meta-analysis evaluating the diagnostic accuracy of Western blot showed a pooled sensitivity of $0.70(95 \%$ confidence interval [CI], 0.59-0.80) and specificity of 0.98 (95\% CI, 0.87-1.00) [14]. Patients also frequently undergo series of tests to rule out common autoimmune diseases. Common lab tests include ESR, CRP, ANA, Rheumatoid factor, and complement levels. Workup should also include tests to rule out syphilis. Imaging studies like MRI of the brain and internal auditory canal and PET scan have failed to demonstrate utility in diagnosis though useful in ruling out other organic causes for hearing loss like acoustic neuroma, intracranial metastases, demyelinating diseases etc. In some cases, MRI may show enhancement of vestibular and cochlear elements.

Prompt and early treatment has a significant impact as AIED is one of the few potentially reversible causes of hearing loss. The most widely used and effective treatment modality in AIED is corticosteroid therapy. Patients are started on steroids without delay when AIED is suspected as disease is very steroid responsive. The initial dosage regimen is $60 \mathrm{mg}$ or $1 \mathrm{mg} / \mathrm{kg}$ per day of prednisone or 6-methylprednisolone for a period of 4 weeks [15]. Low sodium diet and diuretics are also beneficial in the management [16]. In most steroid responsive patients, the dose can be lowered or tapered off without relapse. Some patients can have symptoms refractory to steroids or have side effects that limit the use of steroids, in which case immune modulating therapies are indicated. Methotrexate is a commonly used immunosuppressant in AIED and Sismanis et al. first reported the usefulness of methotrexate in the improvement of symptoms of aural fullness, tinnitus vertigo and hearing [17]. Though shown to be useful in controlling the vestibular symptoms, methotrexate was found to be less effective in maintaining hearing improvement [18]. Other immunosuppressants used includes biologic agents like Infliximab, Etanercept, and Rituximab [19,20]. Intratympanic instillation of medications like steroids has shown promising results [21].There are ongoing clinical trials for drug eluting gels that could be injected into the middle ear space to give sustained drug release. Cochlear implants have also been tried in patients with progressive AIED and they have demonstrated good functional outcomes making it a viable therapeutic option in patients wanting to avoid long term immunosuppression [22].

\section{Conclusion}

Autoimmune inner ear disease (AIED) is a rare distinct cause of idiopathic sensorineural hearing loss. Patients often develop auditory and vestibular symptoms with a rapid progressive fluctuating course. Characteristics of this disease includes responsiveness to steroids and other immunosuppressive agents. There is no gold standard test to aid in the diagnosis of the disease. Prompt and early treatment with immunosuppressants is essential in preventing irreversible hearing loss. The natural history and pathogenesis of the disease still remains to be fully elucidated and further research is needed for a better understanding of this rare disease entity.

\section{References}

1. Bovo R, Ciorba A, Martini A (2009) The diagnosis of autoimmune inner ear disease: evidence and critical pitfalls. Eur Arch Otorhinolaryngol 266: 37-40. [Crossref]
2. McCabe BF (1979) Autoimmune sensorineural hearing loss. Ann Otol Rhinol Laryngol88: 585-589. [Crossref]

3. TomiyamaS, Harris JP (1987)The role of the endolymphatic sac in inner ear immunity Acta Otolaryngol 103: 182-188. [Crossref]

4. Takahashi M, Harris JP (1988) Anatomic distribution and localization of immunocompetent cells in normal mouse endolymphatic sac. Acta Otolaryngol 106: 409-416. [Crossref]

5. Gong SS, Yu DZ, Wang JB (2002) Relationship between three inner ear antigens with different molecular weights and autoimmune inner ear disease. Acta Otolaryngol 122: 5-9. [Crossref]

6. Lobo DR, García-Berrocal JR, Ramírez-Camacho R (2014) New prospects in the diagnosis and treatment of immune-mediated inner ear disease. World J Methodol 4: 91-98. [Crossref]

7. Harris JP, Sharp PA (1990) Inner ear autoantibodies in patients with rapidly progressive sensorineural hearing loss. Laryngoscope 100: 516-524. [Crossref]

8. Moscicki RA, San Martin JE, Quintero CH, Rauch SD, Nadol JB Jr, et al. (1994) Serum antibody to inner ear proteins in patients with progressive hearing loss. Correlation with disease activity and response to corticosteroid treatment. JAMA 272: 611-616. [Crossref]

9. Billings PB, Keithley EM, Harris JP (1995) Evidence linking the 68 kilodalton antigen identified in progressive sensorineural hearing loss patient sera with heat shock protein 70. Ann Otol Rhinol Laryngol 104: 181-188. [Crossref]

10. Hughes GB, Barna BP, Kinney SE, Calabrese LH, Nalepa NJ (1988) Clinical diagnosis of immune inner-ear disease. Laryngoscope 98:251-253. [Crossref]

11. Loveman DM, de Comarmond C, Cepero R, Baldwin DM (2004)Autoimmune sensorineural hearing loss: clinical course and treatment outcome. Semin Arthritis Rheum 34:538-543. [Crossref]

12. Dayal VS, Ellman M, Sweiss N (2008) Autoimmune inner ear disease: clinical and laboratory findings and treatment outcome. J Otolaryngol Head Neck Surg 37: 591596. [Crossref]

13. Broughton SS, Meyerhoff WE, Cohen SB (2004) Immune-mediated inner ear disease: 10-year experience. Semin Arthritis Rheum 34: 544-548. [Crossref]

14. Ianuale C, Cadoni G, De Feo E, Liberati L, Simo RK, et al. (2013) A systematic review and meta-analysis of the diagnostic accuracy of anti-heat shock protein 70 antibodies for the detection of autoimmune hearing loss. Otol Neurotol 34: 214-219. [Crossref]

15. Ruckenstein MJ (2004) Autoimmune inner ear disease. Curr Opin Otolaryngol Head Neck Surg 12: 426-430. [Crossref]

16. Rahman MU, Poe DS, Choi HK (2001) Autoimmunevestibulo-cochlear disorders. Curr Opin Rheumatol 13: 184-189. [Crossref]

17. Sismanis A, Thompson T, Willis HE (1994) Methotrexate therapy for autoimmune hearing loss: a preliminary report. Laryngoscope 104: 932-934. [Crossref]

18. Harris JP, Weisman MH, Derebery JM, Espeland MA, Gantz BJ, et al. (2003) Treatment of corticosteroid-responsive autoimmune inner ear disease with methotrexate: a randomized controlled trial. JAMA 290: 1875-1883. [Crossref]

19. Cohen S, Roland P, Shoup A, Lowenstein M, Silverstein H, et al. (2011) A pilot study of rituximab in immune-mediated inner ear disease. Audiol Neurootol 16: 214-221. [Crossref]

20. Matteson EL, Choi HK, Poe DS, Wise C, Lowe VJ, et al. (2005) Etanercept therapy for immune-mediated cochleovestibular disorders: a multi-center, open-label, pilot study. Arthritis Rheum 53: 337-342. [Crossref]

21. Haynes DS, O'Malley M, Cohen S, Watford K, Labadie RF (2007) Intratympanic dexamethasone for sudden sensorineural hearing loss after failure of systemic therapy. Laryngoscope 117: 3-15. [Crossref]

22. Aftab S, Semaan MT, Murray GS, Megerian CA (2010) Cochlear implantation outcomes in patients with autoimmune and immune-mediated inner ear disease. Otol Neurotol 31: 1337-1342. [Crossref]

Copyright: (C2016 Panikkath D. This is an open-access article distributed under the terms of the Creative Commons Attribution License, which permits unrestricted use, distribution, and reproduction in any medium, provided the original author and source are credited. 